\title{
Clinical impact of newly developed atrial fibrillation complicated with longstanding ventricular fibrillation during left ventricular assist device support: A case report
}

\author{
Chie Bujo ${ }^{1}$, Eisuke Amiya ${ }^{1 *}$ D, Masaru Hatano ${ }^{1,3}$, Masaki Tsuji ${ }^{1}$, Hisataka Maki ${ }^{1}$, Yumiko Hosoya ${ }^{1,3}$, Emi Fujii ${ }^{1}$, \\ Tatsuya Kamon ${ }^{1}$, Toshiya Kojima', Kan Nawata², Osamu Kinoshita², Mitsutoshi Kimura², Minoru Ono ${ }^{2}$ and \\ Issei Komuro ${ }^{1}$
}

\begin{abstract}
Background: Continuous-flow left ventricular assist devices (LVADs) improve survival and morbidity in patients with stage D heart failure. Management of LVADs for longer durations is necessary in some clinical settings, and a better understanding of the hemodynamics of patients using LVADs is warranted. Arrhythmia, including atrial (AA) and ventricular (VAs) arrhythmias, is a modifying factor of hemodynamics that is highly prevalent among patients with LVADs. However, the clinical impact of arrhythmias in various clinical settings in patients with LVAD, in which the hemodynamic load is likely to present as worsening of right heart failure, remains to be completely elucidated.

Case presentation: We describe the case of a patient under sustained ventricular fibrillation for extraordinarily long duration who was stabilized using LVAD support and in whom newly developed atrial fibrillation led to a significant worsening of right heart failure while using an LVAD.
\end{abstract}

Conclusion: This case demonstrates the substantial clinical impact of AAs in the management of right heart failure using an LVAD.

Keywords: Left ventricular assist device, Right heart failure, Ventricular fibrillation, Atrial fibrillation

\section{Background}

In patients with medically intractable heart failure, continuous-flow left ventricular assist devices (LVADs) improve quality of life as well as survival and morbidity rates compared with conventional medical therapy [1]. Although LVAD use improves the hemodynamic derangement, such as decreased output, triggered by impaired left ventricular systolic function or mitral regurgitation, atrial arrhythmias (AAs) and ventricular arrhythmias (VAs), both highly prevalent in LVAD patients, are considered as poor prognostic factors [2]. During ventricular fibrillation (VF), it is sometimes possible to maintain hemodynamic stability using LVADs,

\footnotetext{
* Correspondence: amiyae-tky@umin.ac.jp

${ }^{1}$ Department of Cardiovascular Medicine, Graduate School of Medicine, The University of Tokyo, Hongo 7-3-1, Bunkyo-ku, Tokyo 113-8655, Japan Full list of author information is available at the end of the article
}

however, the hemodynamic characteristics of these arrhythmias under LVADs have not been fully elucidated. In particular, the association between right heart failure and the development of arrhythmias in patients with LVADs remains unclear. We report the case of a patient with sustained VF for 3 years under LVAD support who had worsening of heart failure with new onset of atrial fibrillation (AF).

\section{Case presentation}

A 47-year-old male developed heart failure due to dilated cardiomyopathy 12 years ago. A cardiac resynchronization therapy-defibrillator (CRT-D; Medtronic Viva XT CRT-D; AAI 60) was implanted due to VF 7 years ago, and as a bridge to transplantation, a HeartMate $\mathrm{II}^{\circ}$ LVAD was implanted 4 years ago. No arrhythmia developed immediately after LVAD implantation; thus, his

(c) The Author(s). 2019 Open Access This article is distributed under the terms of the Creative Commons Attribution 4.0 International License (http://creativecommons.org/licenses/by/4.0/), which permits unrestricted use, distribution, and reproduction in any medium, provided you give appropriate credit to the original author(s) and the source, provide a link to the Creative Commons license, and indicate if changes were made. The Creative Commons Public Domain Dedication waiver (http://creativecommons.org/publicdomain/zero/1.0/) applies to the data made available in this article, unless otherwise stated. 
CRT-D shock therapy was turned off immediately after LVAD implantation. At the time of LVAD implantation, his transthoracic echocardiographic study showed a significant decrease in the left ventricular (LV) contractility (ejection fraction; 13\%), dilation of left ventricle (51 mm in diastole) and trivial aortic regurgitation (AR) without opening of aortic valve but right ventricular (RV) contraction had maintained well relatively (RV fractional area change; 33\%).

Eight months after LVAD implantation, the patient developed palpitations and was admitted to our hospital due to repeated VAs necessitating electrical defibrillation. Echocardiography showed the left ventricle diameter did not change, whereas right ventricle volume was slightly enlarged. The repeated VAs were also refractory to various anti-arrhythmic agents, including amiodarone, nifekalant, mexiletine and lidocain, with eventual progression to sustained VF. The hemodynamic compromise due to sustained VF resulted in liver congestion, which was alleviated with a phosphodiesterase type 5 inhibitor, diuretics, and rotation speed optimization (from 8800 to $9600 \mathrm{rpm}$ ). These interventions reduced organ dysfunction, suggesting that minimum-required perfusion to vital organs was maintained even under sustained VF. The patient was followed up on an outpatient basis thereafter.

Approximately 2 years after the development of sustained VF, paroxysmal AF was detected on the monitoring records of CRT-D, with a gradually increasing frequency.
After 3 years of sustained VF, the patient was readmitted to our hospital due to worsening of symptoms associated with right heart failure and liver congestion (total bilirubin, $3.9 \mathrm{mg} / \mathrm{dl}$ ). Although his electrocardiogram remained sustained VF (Fig. 1), the CRT-D revealed conversion of the sinus or atrial pacing rhythm to persistent AF. Transthoracic echocardiography revealed that fibrillation of the atrium resulted in the disappearance of not only the mitral flow but also the RV outflow tract doppler flow by the atrial kick (Fig. 2, Additional file 1, 2, 3, 4, 5 and 6). Under sustained VF, RV cardiac output is greatly dependent on atrial kick in which the contribution of atrial kick extraordinary enhanced. The hemodynamic study indicated that the pressure wave from the right atrium (RA) to the right ventricle was significantly flattened resulting that the pulmonary artery pulsatility index, which is defined as the ratio of pulmonary artery pulse pressure to right atrial pressure, was markedly decreased. It suggested a marked reduction in blood flow induced by RA contraction in persistent AF as opposed to sinus rhythm (Fig. 3). As the incremental rotation speed lead to an increase in RA pressure (RAP) from $19 \mathrm{mmHg}$ to $22 \mathrm{mmHg}$, the rotation speed was set to $9600 \mathrm{rpm}$ and the right heart congestion was treated with additional diuretics insufficiently and the enhanced level of total bilirubin was prolonged. Until four months later, heart failure was gradually improved and the level of total bilirubin decreased below $2 \mathrm{mg} / \mathrm{dl}$. The monitoring records of CRT-D revealed the recovery of sinus rhythm during previous four months (Fig. 4). Although he

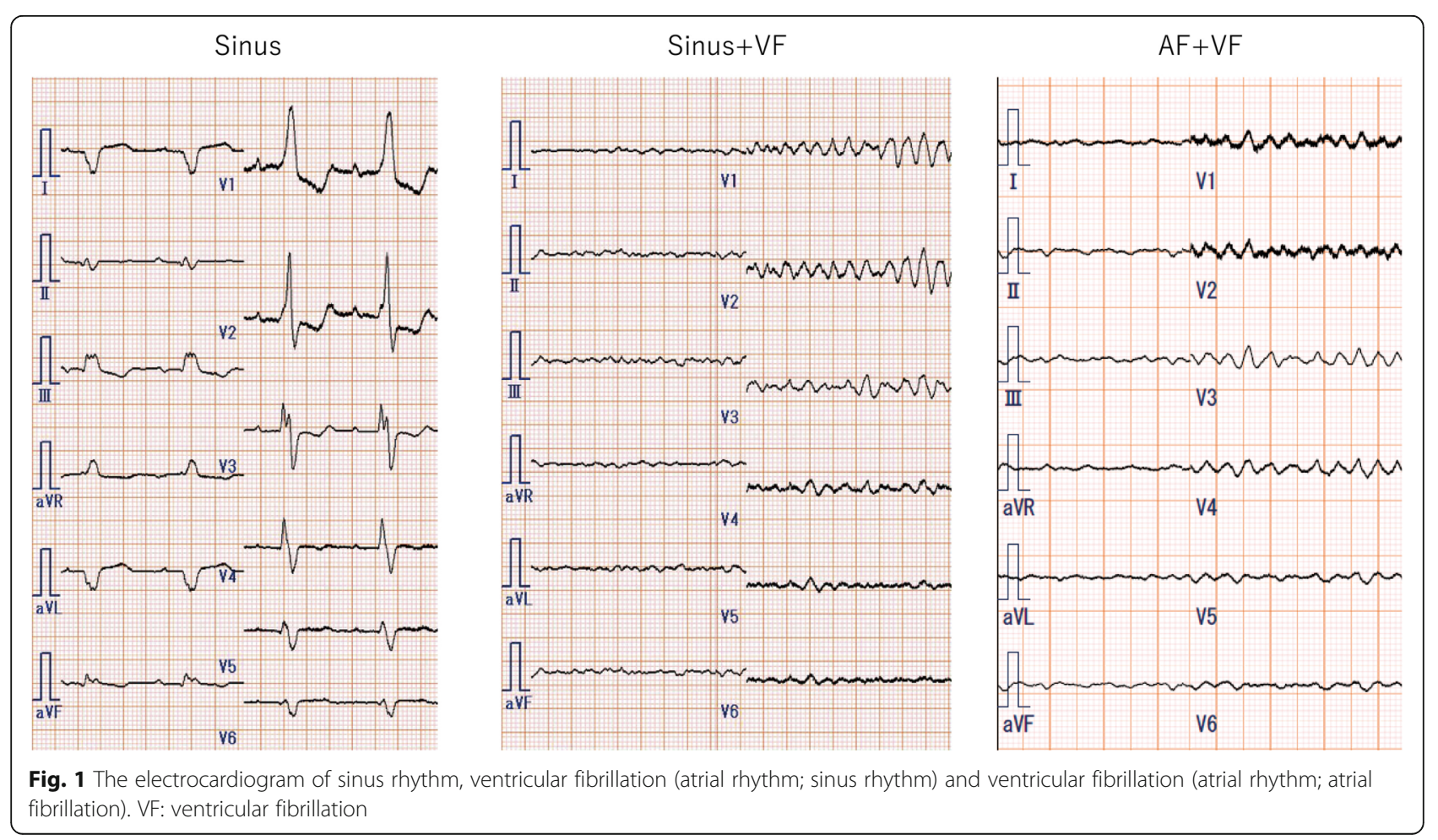



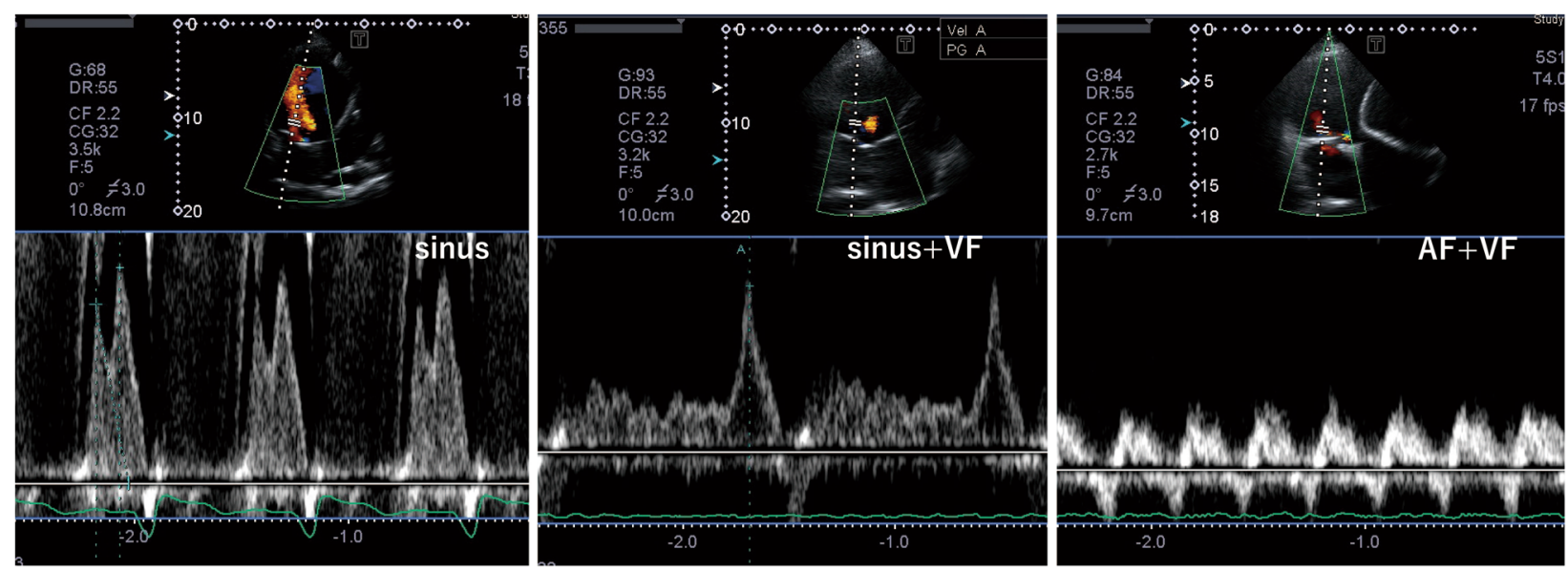

Fig. 2 Pulse-wave Doppler showing transmitral flow wave in each rhythm. Normal mitral valve E and A waves were observed in sinus rhythm, whereas only mitral valve A wave could be observed during sinus rhythm and ventricular fibrillation (sinus + VF). During atrial and ventricular fibrillation $(A F+V F)$, no obvious E or A waves were observed; however, small fibrillating waves were detected. Abbreviations: AF: atrial fibrillation, VF: ventricular fibrillation

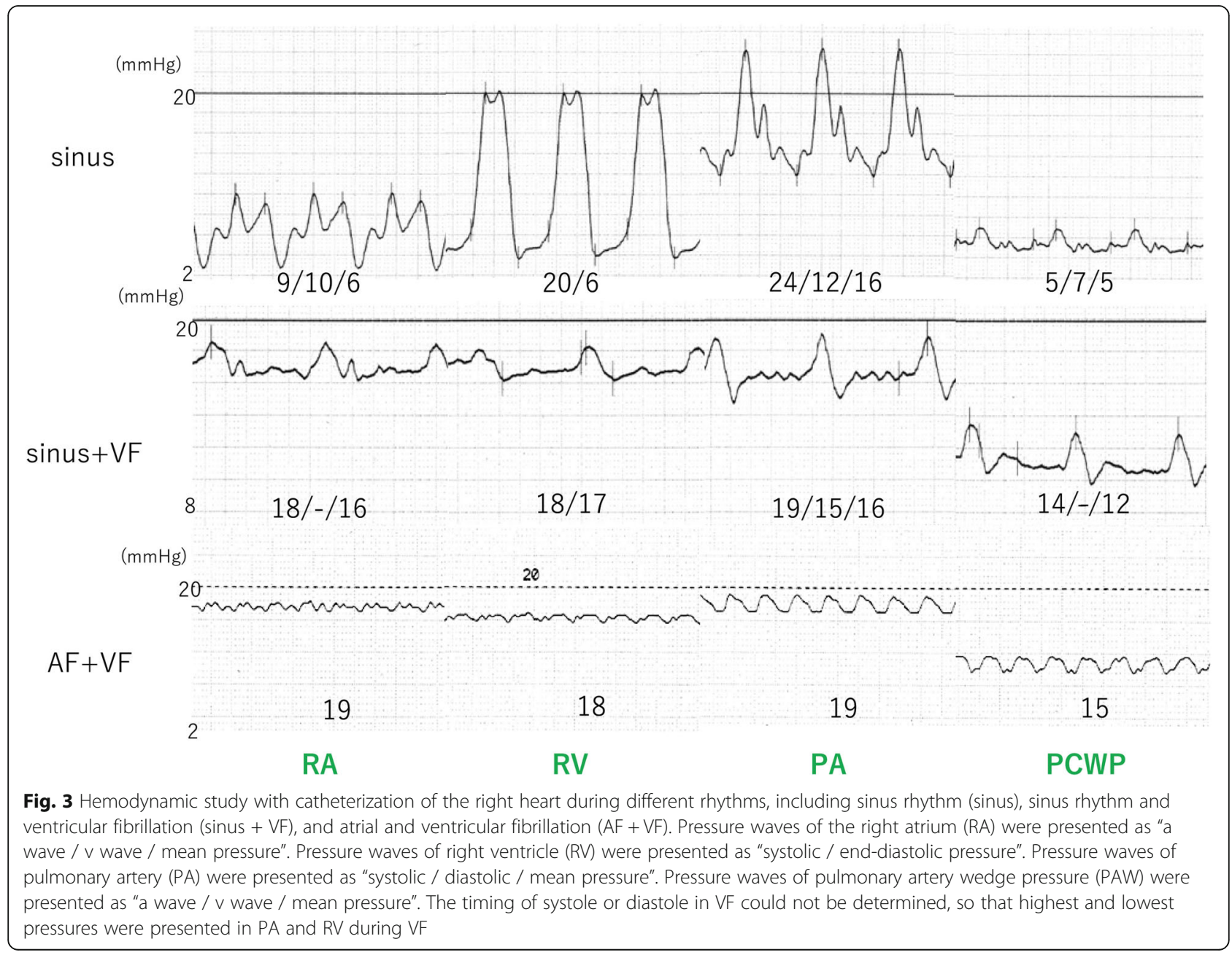




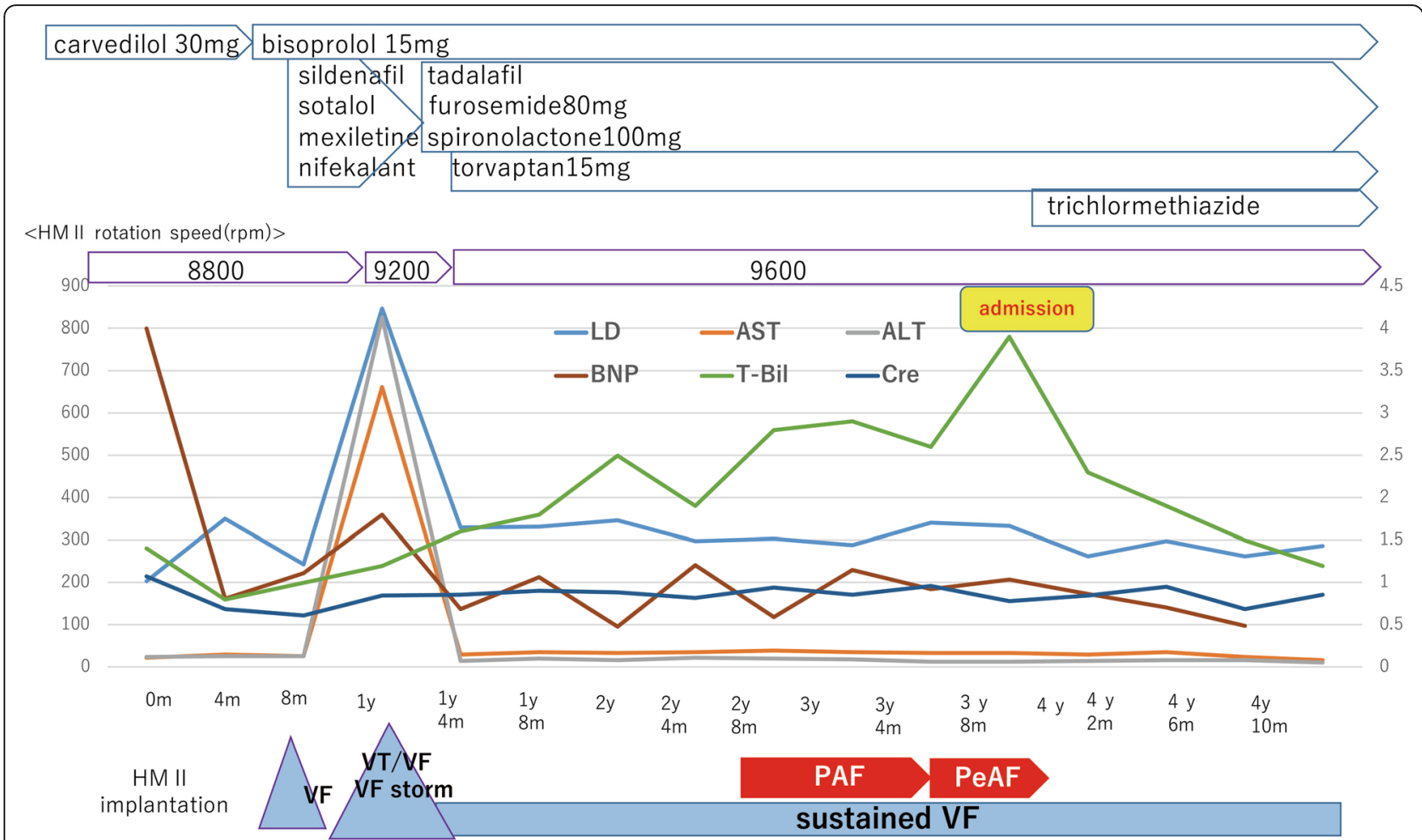

Fig. 4 Time course of laboratory data including lactate dehydrogenase (LD), aspartate aminotransferase (AST), alanine aminotransferase (ALT), Bnatriuretic peptide (BNP), total bilirubin (T-Bil), and creatinine (Cre) after LVAD implantation. Medications were recorded and each arrhythmic event was described. Abbreviations: VT; ventricular tachycardia, VF; ventricular fibrillation, PAF; paroxysmal atrial fibrillation, PeAF; persistent atrial fibrillation, HMIl; HeartMate II

had remained sustained VF, the recovery of sinus rhythm finely corresponded to the improvement of heart failure and the level of his total bilirubin decreased to $1.2 \mathrm{mg} / \mathrm{dl}$. It intensely corroborated the contribution of persistent $\mathrm{AF}$ on the worsening of right heart failure.

\section{Discussion and conclusions}

Both AAs and VAs are common in patients with severe heart failure under LVAD support; furthermore, VAs have been reported in about $20 \%$ of LVADs patients [3], with some of them being refractory to various therapies. AAs are also common in patients under LVAD support and are associated with increased readmission and mortality rates [4]. In Japan, long-term LVAD use has become a necessity due to an extreme shortage of donor hearts, requiring a more efficient management of such arrhythmia-related issues [5].

We have reported the case of a patient who developed VAs 8 months after LVAD implantation and sustained VF for the following 4 years. To date, there have been several case reports revealing the tolerability of sustained VF under LVADs; furthermore, we have also experienced some cases of sustained VF without progressive organ dysfunction [5]. However, hemodynamics is not preserved in some patients under sustained VF, and the clinical factors that determine the tolerability of sustained VF remain unknown [6]. The patient in the present case tolerated sustained VF for the longest duration about 3 years reported in the published literature. However, after the onset of AF, the patient gradually developed right heart failure and it was worsened further after he developed persistent AF. The current case suggested the hemodynamic status of VF under LVAD might be determined by other surrounding factors such as atrial function and the change of sinus or atrial pacing rhythm to AF led to the disappearance of regular RA contractions. Consequently, the atrial wave of RAP disappeared and the pressure wave pattern of RAP and RV completely flattened. The subsequent reductions in the RA and RV outputs supposedly led to a worsening of the patient's right heart failure.

Right atrial contraction contributes to approximately $15-30 \%$ of the RV stroke volume; however, its contribution differs across patients with heart failure. The development of AF generally decreases the cardiac output [7], whereas the derangement of AF in cardiac output is somewhat counteracted under LVAD support. Enriquez et al. have demonstrated no significant differences in peak $\mathrm{VO}_{2}$ during a cardiopulmonary exercise test between patients with and without AF under LVAD 
support [2]. Conversely, the incidence of heart failure hospitalization has reportedly increased due to AF, suggesting that the right side of the heart remains sensitive to the hemodynamic effects of AF. A small case series demonstrated an improvement in right heart failure after catheter ablation of AF in patients with LVADs [8]; therefore, rhythm control may be an important strategy for managing right heart failure for this group of patients. If it was difficult to control of our patient's right heart failure, we considered the catheter ablation of AF. In the present case, the pulmonary artery pulsatility index, which was reported to correspond to RV stroke volume [9], was somewhat maintained in sinus rhythm even under the sustained VF, whereas it significantly decreased by the development of AF. Several clinical conditions of right heart failure enhance the impact of right atrial function [10]. D'Alto et al. have also demonstrated the enhanced impact of right atrial function in idiopathic pulmonary artery hypertension [11]. Specifically, the contribution of AAs is significantly enhanced in sustained VF, in which the fibrillating ventricle has minimal contribution to the systemic output, and the coexistence of AAs and VAs may be critical. The current case highlights the significance of right atrial function as a determinant of hemodynamics in patients with sustained VF under LVAD support and suggests a non-invasive approach for the treatment of heart failure.

According to the risk of thromboembolism, it is complicated in patients with AF under LVAD support. There was a report of thromboembolism risk in patients with AF under LVAD [12], whereas a recent meta-analysis exhibited the presence of AF did not increase the risk of thromboembolism [13].

This case also emphasizes the difficulty of diagnosing AF under sustained VF. The findings on electrocardiogram of patients under sustained VF remain unchanged when AF is newly developed. Therefore, the detection of AF may be difficult, and monitoring of device records, mitral flow visualization using echocardiography or visualization of pressure wave by Swan-Ganz catheter is required. The occurrence of newly developed AF under sustained VF is a rare case; however, the understanding of the hemodynamic effect of these arrhythmias under LVAD support is much informative for future cases of LVAD support.

Indeed, AF might be a sign or symptom of a deterioration of HF, however, the risk of new development of AF is complicated under LVAD. For instance, Deshmukh A et al. demonstrated paroxysmal AF was improved after LVAD implantation due to improvement of electroanatomical remodeling [14]. On the other hand, LVAD related complications such as $\mathrm{AR}$ and right heart failure might also impose the excessive burden to atrium leading to AF progression. The determining factor of $\mathrm{AF}$ progression in the current study was beyond the scope of the current case report.

We report the case of a patient with sustained VF for 3 years under LVAD support who had worsening of heart failure with new onset of AF. The hemodynamic characteristics of stabilized sustained VF under LVADs have not been fully elucidated, however this case showed the contribution of AAs is significantly enhanced in sustained VF.

\section{Additional files}

Changes in the left ventricular (LV) and left atrial (LA) contraction as well
as in blood flow in the tricuspid valve during each rhythm [1-2: sinus
rhythm, 3-4: sinus rhythm and ventricular fibrillation (sinus + VF), 5-6:
atrial fibrillation and ventricular fibrillation (AF + VF)]. In the sinus rhythm,
LV and LA contracted regularly in turn (1) and rhythmic blood flow in the
tricuspid valve was observed (2). There was no significant tricuspid valve
regurgitation. In the sinus + VF rhythm, LA contracted regularly, whereas
no visible contractions were observed by fibrillating LV (3, 4). The regular
mitral and tricuspid valve movements were also observed. In the AF + VF
rhythm, no regular contractions of LV and LA were observed (5) and
blood flow to and from the tricuspid valve was detected (6). The regular
mitral and tricuspid valve movements could not be observed.
Additional file 1 (MOV $108 \mathrm{~kb}$ )
Additional file 2 (MOV $87 \mathrm{~kb}$ )
Additional file 3 (MOV $148 \mathrm{~kb}$ )
Additional file 4 (MOV $147 \mathrm{~kb}$ )
Additional file 5 (MOV $133 \mathrm{~kb}$ )
Additional file 6 (MOV $128 \mathrm{~kb}$ )

\section{Abbreviations}

AA: Atrial arrhythmia; AF: Atrial fibrillation; AR: Aortic regurgitation; LV: Left ventricle; LVAD: Left ventricular assist devices; RA: Right atrium; RAP: Right atrial pressure; RV: Right ventricle; VF: Ventricular fibrillation

\section{Acknowledgements}

None.

\section{Authors' contributions}

EF, TK1, EA, HM, MH, OK and MK followed up the patients. CB and EA drafted the manuscript and performed the literature review. MT, YH, TK2, NK, MO and IK critically revised the manuscript. All authors have read and approved the final manuscript.

\section{Funding}

This research did not receive any specific grant from funding agencies in the public, commercial, or not-for-profit sectors.

Availability of data and materials

Not applicable.

Ethics approval and consent to participate

Not applicable.

Consent for publication

Written informed consent for publication of the clinical detailed was obtained from the patient.

Competing interests

The authors declare that they have no competing interests. 


\section{Author details}

'Department of Cardiovascular Medicine, Graduate School of Medicine, The University of Tokyo, Hongo 7-3-1, Bunkyo-ku, Tokyo 113-8655, Japan. ${ }^{2}$ Department of Cardiac Surgery, Graduate School of Medicine, The University of Tokyo, Tokyo, Japan. ${ }^{3}$ Department of Therapeutic Strategy for Heart Failure, Graduate School of Medicine, The University of Tokyo, Tokyo, Japan.

Received: 25 February 2019 Accepted: 11 June 2019

Published online: 21 June 2019

\section{References}

1. Rose EA, Gelijns AC, Moskowitz AJ, Heitjan DF, Stevenson LW, Dembitsky W, et al. Long-term use of a left ventricular assist device for end-stage heart failure. N Engl J Med. 2001;345:1435-43.

2. Enriquez AD, Calenda B, Gandhi PU, Nair AP, Anyanwu AC, Pinney SP. Clinical impact of atrial fibrillation in patients with the HeartMate II left ventricular assist device. J Am Coll Cardiol. 2014;64:1883-90.

3. Bedi M, Kormos R, Winowich S, McNamara DM, Mathier MA, Murali S. Ventricular arrhythmias during left ventricular assist device support. Am J Cardiol. 2007;99:1151-3.

4. Deshmukh A, Kim G, Burke M, Anyanwu E, Jeevanandam V, Uriel N, et al. Atrial arrhythmias and electroanatomical remodeling in patients with left ventricular assist devices. J Am Heart Assoc. 2017;6:e005340.

5. Naito N, Kinoshita O, Ono M. Prolonged left ventricular assist device support (18months) in refractory ventricular fibrillation. J Heart Lung Transplant. 2014;33:772-3.

6. Garan AR, Yuzefpolskaya M, Colombo PC, Morrow JP, Te-Frey R, Dano D, et al. Ventricular arrhythmias and implantable cardioverter-defibrillator therapy in patients with continuous-flow left ventricular assist devices: need for primary prevention? J Am Coll Cardiol. 2013;61:2542-50.

7. Takano N, Amiya E, Oguri G, Nakayama A, Taya M, Nakajima T, et al. Influence of atrial fibrillation on oxygen uptake and exercise tolerance in cardiovascular patients; close association with heart rate response. Int J Cardiol Heart Vasc. 2019;22:84-91.

8. Maury P, Delmas C, Trouillet C, Slaughter MS, Lairez O, Galinier M, et al. First experience of percutaneous radio-frequency ablation for atrial flutter and atrial fibrillation in a patient with HeartMate II left ventricular assist device. J Interv Card Electrophysiol. 2010;29:63-7.

9. Kang $G, H a$ R, Banerjee D. Pulmonary artery pulsatility index predicts right ventricular failure after left ventricular assist device implantation. J Heart Lung Transplant. 2016;35:67-73.

10. Hawkins RB, Mehaffey JH, Guo A, Charles EJ, Speir AM, Rich JB, et al. Postoperative atrial fibrillation is associated with increased morbidity and resource utilization after left ventricular assist device placement. J Thorac Cardiovasc Surg. 2018 Oct;156(4):1543-9.

11. D'Alto M, D'Andrea A, Di Salvo G, Scognamiglio G, Argiento P, Romeo E, et al. Right atrial function and prognosis in idiopathic pulmonary arterial hypertension. Int J Cardiol. 2017;248:320-5.

12. Enriquez AD, Calenda B, Gandhi PU, Nair AP, Anyanwu AC, Pinney SP. Clinical impact of atrial fibrillation in patients with the HeartMate II left ventricular assist device. J Am Coll Cardiol. 2014;64(18):1883-90.

13. Kittipibul V, Rattanawong P, Kewcharoen J, Chongsathidkiet P, Vutthikraivit W, Kanjanahattakij N. Atrial fibrillation is not associated with thromboembolism in left ventricular assist device patients: a systematic review and meta-analysis. ASAIO J. 2018; epub ahead of print.

14. Deshmukh A, Kim G, Burke M, Anyanwu E, Jeevanandam V, Uriel N, et al. Atrial arrhythmias and electroanatomical remodeling in patients with left ventricular assist devices. J Am Heart Assoc. 2017;6:e005274.

\section{Publisher's Note}

Springer Nature remains neutral with regard to jurisdictional claims in published maps and institutional affiliations.

Ready to submit your research? Choose BMC and benefit from:

- fast, convenient online submission

- thorough peer review by experienced researchers in your field

- rapid publication on acceptance

- support for research data, including large and complex data types

- gold Open Access which fosters wider collaboration and increased citations

- maximum visibility for your research: over $100 \mathrm{M}$ website views per year

At BMC, research is always in progress.

Learn more biomedcentral.com/submissions 